\title{
Prevención de la infección por el virus de la inmunodeficiencia humana (VIH) en Colombia: brechas y realidades*
}

\section{Prevention of Human Immunodeficiency Virus (HIV) Infection in Colombia: Gaps and Realities}

\section{Prevenção da infeção pelo vírus da imunodeficiếncia humana (VIH) na Colômbia: fendas e realidades}

Fecha de recepción: 20-04-2016 Fecha de aceptación: 20-05-2017 Disponible en línea: 30-05-2017 doi:10.11144/Javeriana.rgps16-33.pivi

Cómo citar este artículo:

Mora-Rojas RB, Alzate-Posada ML, Rubiano-Mesa YL. Prevención de la infección por el virus de la inmunodeficiencia humana en Colombia: brechas y realidades. Rev Gerenc Polít Salud. 2017; 16 (33): 19-34. https:// doi.org/10.11144/Javeriana.rgps16-33.pivi

\author{
Ruth Beatriz Mora-Rojas** \\ Marta Lucia Alzate-Posada*** \\ Yurian Lida Rubiano-Mesa****
}

\footnotetext{
* Artículo de revisión. Ensayo presentado el 13 de abril de 2016 como examen de calificación para optar al grado de candidatura a doctor en Salud Pública. La financiación estuvo a cargo de Colciencias (2014-2017).

** Enfermera, candidata a doctor en Salud Pública de la Universidad Nacional de Colombia, profesora de la Fundación Universitaria del Área Andina, magistrada del Tribunal Nacional Ético de Enfermería, 2010-2014 y 2014-2018. Dirección de correspondencia: Calle 25 68B - 47 Apto. 403 Interior 2, Bogotá, Colombia. Correo electrónico: rbmorar@unal.edu.co ruthbeatrizmora@hotmail.com

*** Enfermera, Ph. D., doctora en Salud Pública, profesora asociada de la Facultad de Enfermería, profesora del doctorado Interfacultades de Salud Pública, Universidad Nacional de Colombia. Directora de Bienestar Universitario de la Universidad Nacional de Colombia. Correo electrónico: mlalzatep@unal.edu.co

***** Enfermera, Ph. D., doctora en Ciencias Sociales, Niñez y Juventud, profesora de la Facultad de Enfermería de la Universidad Nacional de Colombia, actual vicedecana de dicha facultad. Correo electrónico: ylrubianom@unal.edu.co
} 


\section{Resumen}

Este artículo pretende analizar cómo ha sido la prevención de la infección por el VIH en Colombia, desde la década de los ochenta hasta la actualidad, por ser una de las principales medidas para el control de la infección. Presenta el contexto epidemiológico en el mundo y en Colombia, el desarrollo de la prevención de la infección y finaliza con reflexiones sobre brechas y realidades de lo que ha caracterizado la prevención del VIH en nuestro medio. Metodología: se analizaron documentos y artículos científicos en español, inglés y portugués del año 2000 al 2016, disponibles en PubMed Lilacs, Ebsco, Medline, Justor y Scielo. Se revisaron setenta y cinco artículos y se seleccionaron veintinueve. Conclusiones: la prevención del VIH debe avanzar del enfoque asistencialista a políticas que promuevan el desarrollo humano, social y económico, sin olvidar que la población en general se encuentra en riesgo de adquirir la infección.

Palabras clave: Prevención; VIH, SIDA; salud pública; vulnerabilidad; discriminación

\section{Abstract}

This article aims to analyze how the prevention of HIV infection in Colombia-from the 1980s until now- has taken place, given that it is one of the main measures to control infection. We present the epidemiological context in the world and in Colombia, the development of the prevention of infection, and conclude with some reflections on the gaps and realities of what has characterized HIV prevention in our environment. Methodology: we analyzed papers and scientific articles in Spanish, English, and Portuguese from 2000 to 2016, available in PubMed Lilacs, Ebsco, Medline, Justor, and Scielo. Seventy-five articles were reviewed out of which twenty-nine selected. Conclusions: HIV prevention must move from the assistance-based approach to policies that promote human, social, and economic development, without leaving aside the fact that the general population is at risk of acquiring the infection.

Keywords: Prevention; HIV; AIDS; health public; vulnerability; discrimination

\section{Resumo}

Este artigo visa analisar como foi a prevenção da infeção pelo VIH na Colômbia, desde a década de oitenta para hoje, por ser uma das principais medidas para o controle da infeção. Apresenta o contexto epidemiológico no mundo e na Colômbia, o desenvolvimento da prevenção da infeção e finda com reflexões sobre fisgas e realidades do que já caracterizou a prevenção do VIH no nosso meio. Metodologia: analisaram-se documentos e artigos científicos em espanhol, inglês e português do ano 2000 para 2016, disponíveis em PubMed Lilacs, Ebsco, Medline, Justor e Scielo. Revisaram-se setenta e cinco artigos e selecionaram-se vinte e nove. Conclusões: a prevenção do VIH deve avançar do enfoque assistencialista para políticas promovendo o desenvolvimento humano, social e económico, sem esquecer que a população em geral encontra-se em risco de adquirir a infeção.

Palavras chave: Prevenção; VIH; SIDA; saúde pública; vulnerabilidade; discriminação 


\section{Introducción}

La infección por el virus de la inmunodeficiencia humana (VIH) se considera un grave problema de salud pública por la magnitud de la infección como epidemia de orden global y local, así como por las repercusiones en la salud individual y poblacional. Afecta negativamente aspectos biológicos, psicológicos, sociales y económicos de quien la padece, de la familia y de la sociedad. En la dimensión biológica pueden presentarse enfermedades oportunistas; en el plano psicológico y social aparecen reacciones de temor y culpa. El estigma y la discriminación hacia los afectados pueden ocasionar abandono por parte de la familia y los amigos o ser excluidos y censurados por otros miembros de la sociedad. En lo económico, produce alto impacto debido a la complejidad y cronicidad de la enfermedad, los costos de los servicios de salud, los costos que debe asumir la familia, la pérdida laboral, el abandono de la actividad económica y la disminución de la población en edad productiva $(1,2)$.

Estos aspectos inciden en el bienestar y la calidad de vida del individuo que vive con VIH, en cuanto a lograr su proyecto de vida. La infección altera las dinámicas familiares de quien la padece $(1,3)$ y de la sociedad en la que vive. A pesar de que la investigación científica realizada a la fecha evidencia avances positivos en la prevención, el diagnóstico y el tratamiento de la infección, se reconoce que sus resultados no son suficientes para garantizar el control de la epidemia (4).

Por lo anterior, este artículo tiene como objetivo revisar y analizar cómo ha sido la prevención de la infección por el VIH, por ser esta una de las principales estrategias para el control de la infección. En la primera parte se presenta el contexto epidemiológico de la infección del VIH en el mundo y en Colombia, se continúa con el desarrollo de la prevención de la infección para, finalmente, llegar a plantear algunas reflexiones sobre posibles brechas y realidades de la prevención del VIH en nuestro medio.

\section{Contexto mundial y nacional de la infección por el VIH}

En el mundo, según el informe del Programa de Naciones Unidas sobre el VIH/SIDA, Onusida 2015, dos millones de personas se infectaron con el VIH al final de 2014 y 1.2 millones murieron como consecuencia del SIDA (5). Mientras hay una tendencia al descenso de nuevas infecciones, la mortalidad disminuye y la prevalencia aumenta (5), como resultado de las nuevas infecciones y de los efectos positivos de la terapia antirretroviral (TAR) (6), la cual permite a la población afectada por esta infección prolongar su supervivencia y llevar una vida normal y productiva. Esto demuestra que, a pesar de los avances en el control de la infección por el VIH, esta se transformó en una enfermedad crónica, compleja, evitable y controlable. Por ello, es necesario continuar haciendo esfuerzos para lograr su control y eliminación.

Según la literatura, la población joven y marginada continúa siendo el centro de la epidemia, ligada a comportamientos de riesgo individuales, como el inicio temprano de su vida sexual sin protección, el uso de drogas, las condiciones de desigualdad social, el estigma, la discriminación, la violencia y a los determinantes sociales de la salud (DSS) y económicos como la pobreza y la baja escolaridad, que potencian la transmisión de la infección y favorecen la propagación de la epidemia $(7,8)$. Un panorama que refleja, en parte, la importancia de la prevención en VIH.

Colombia ha sido clasificada como un país con una epidemia concentrada, considerando que la población de mayor vulnerabilidad es la 
conformada por los hombres que tienen sexo con hombres (HSH), las mujeres transgénero, los habitantes de calle, los usuarios de drogas, las y los trabajadores sexuales y población privada de la libertad (PPL) $(9,10)$, sin desconocer las poblaciones de adolescentes, mujeres y madres gestantes.

Es decir, la infección por VIH afecta principalmente a poblaciones en condiciones socioeconómicas desfavorables, con mayor vulneración a sus derechos y necesidades insatisfechas. Razones asociadas, por un lado, con los obstáculos al acceso a la educación, a la información, al diagnóstico y al tratamiento oportuno; pero también al miedo, el estigma y la discriminación de que son objeto las personas afectadas. Por otro lado, el hecho de que la atención brindada por el Estado no logra garantizar la restitución de condiciones dignas para su vivir (11). Esto muestra la actual dinámica socioepidemiológica del VIH/SIDA y, por lo tanto, las estrategias de prevención deben adecuarse y priorizarse para los grupos más vulnerables.

Según el informe de Onusida del año 2014 y el Instituto Nacional de Salud de Colombia, el mayor número de casos de infección se ubica entre los 15 y los 34 años de edad (12,13), es decir, entre la población en edad productiva, en su mayoría hombres, con tendencia al incremento de la transmisión heterosexual en mujeres, por múltiples factores, entre ellos el bajo uso del condón en parejas estables, producto de la cultura machista, la inequidad de género, la violencia, el desplazamiento y la pobreza $(9,14)$. Lo anterior explica la disminución en la razón de masculinidad, que pasa de 10:1 en la década de los noventa a 2.5:1 a finales de 2012 (12,15), con un incremento significativo del número de mujeres con VIH y una alta posibilidad de infectarse en el curso de sus vidas, razón por la cual existe la necesidad de visibilizar las dificultades que viven las mujeres con VIH, para dar respuestas específicas, integrales y oportunas de prevención, diagnóstico y tratamiento.

En el interior del país, la epidemia se comporta de forma diferente, dependiendo de las características culturales y los contextos. Por ejemplo, según el informe nacional del Ministerio de Salud y Protección Social (MSPS) del año 2014, la epidemia crece más rápidamente en la Costa Atlántica, seguida por la Región Pacífica y el Eje Cafetero, con tendencia a la generalización $(9,10)$, si no se buscan intervenciones de reducción de las condiciones de vulnerabilidad que hagan énfasis en los grupos más afectados. Sin embargo, existe una tendencia a la reducción de la mortalidad desde el año 2008 (10), como respuesta a los avances en la prevención en el marco de los derechos humanos (DH), a la atención integral y a la disponibilidad de los antirretrovirales (ARV), acciones claves para cortar la cadena de transmisión y mejorar la calidad de vida de las personas afectadas.

La prevención para el control de la epidemia necesita, por lo tanto, de la cooperación política y financiera de los organismos internacionales y nacionales, así como también de la participación activa de la comunidad y los profesionales de salud para incidir en los DSS que favorecen la propagación del VIH y propender así por la búsqueda de protección social, acceso universal a servicios de información, promoción de la salud sexual y reproductiva (SSR), atención de calidad y tratamiento enfocado en la población afectada.

\section{Desarrollo de la prevención de la infección por VIH}

Dado que la distribución de la vulnerabilidad y el riesgo de adquirir la infección VIH varía de una sociedad a otra, no existe un enfoque único de prevención que resulte eficaz, por lo cual se precisa de diversas maneras de 
percibir, explicar y abordar la problemática, para responder a necesidades particulares de la población, que superen los modelos curativos y en las cuales predominen estrategias de mayor cobertura de la población destinataria, como son las acciones de promoción de la salud (PS) y prevención de la enfermedad (PE) para el control de la infección, en este caso del VIH/SIDA.

E1 VIH, por tratarse de un problema de salud pública, ha sido incluido en las agendas de gobierno y en las políticas públicas, siguiendo los lineamientos de la Organización Mundial de la Salud (OMS), la Organización Panamericana de la Salud (OPS), el Programa de Naciones Unidas (Onusida) y la Asamblea General de Naciones Unidas (Ungass) para la prevención del VIH y la atención de los afectados por la infección, cuyo cometido es detener la propagación del VIH/SIDA, mediante el fortalecimiento de la respuesta del sector salud (pruebas diagnósticas, maximizar la prevención, reforzar y ampliar los servicios sanitarios) (16), sin desconocer las implicaciones sociales, económicas y políticas que inciden en la propagación de la infección. En este marco se presentan los principales enfoques y el desarrollo de la prevención de la infección, desde la década de los ochenta hasta la actualidad.

En la década de los ochenta, ante la epidemia del VIH/SIDA y a partir de la Declaración de Atención Primaria en Salud (APS) en Alma Ata de $1978,{ }^{1}$ se reconoce la salud como un derecho humano fundamental y el derecho de la comunidad a participar en la planificación de la atención en salud. Numerosas organizaciones de la sociedad civil, como la Cruz Roja Internacional, organizaciones no gubernamentales (ONG) y grupos de mujeres, entre otras, se organizaron para ofrecer apoyo

1 En la Conferencia Internacional realizada en Alma Ata, Rusia en 1978 se firmó la Declaración sobre Atención Primaria de la Salud (APS). y asesoría a las personas con VIH/SIDAy a sus familias, y llevar a cabo acciones ligadas a la prevención de la infección en el marco de la APS, con énfasis en la educación comunitaria, la atención y los tratamientos (17).

Por otro lado, la respuesta de las personas afectadas, frente a los primeros reportes de SIDA en población HSH, fue la negación, por el temor a la discriminación y a la misma enfermedad, considerada mortal; simultáneamente, desde la perspectiva institucional, se implementaron las estrategias tradicionales de salud pública centradas en la notificación, el aislamiento y la cuarentena para las infecciones, lo que mostró poca efectividad, por tratarse de métodos coercitivos que, adicionalmente, vulneraban los DH de los afectados (18).

La aplicación de la estrategia de notificación generó el activismo de la comunidad gay, por considerar que se vulneraban sus derechos, lo cual llevó a un abordaje desde el "voluntarismo" - llamado también "excepcionalismo ${ }^{2}$ delVIH”- (19), enfoque que ha caracterizado las políticas en salud pública de la infección, buscando respetar la autonomía y los derechos a la privacidad de las personas afectadas (20). Posteriormente, surgieron los lineamientos normativos de la consejería y consentimiento informado para las pruebas de detección del VIH (19). Esta perspectiva excepcionalista implica la revisión permanente de la implementación de las políticas sanitarias en torno al VIH/SIDA, para adecuarlas a las realidades sociales locales $(19,21)$. Muestra de ello es la necesidad de incluir las pruebas universales de diagnóstico delVIH para las gestantes, con el fin de prevenir la transmisión vertical (20) y proteger los derechos y la salud del binomio madre-hijo.

2 El excepcionalismo propone no aplicar medidas rutinarias que, en la infección por VIH, pueden ser lesivas para la persona afectada en lo psicológico y social (19). 
En la década de los ochenta, la PE se vinculó a la epidemiología tradicional, centrada en la perspectiva de la causalidad y en la identificación y el control de factores de riesgo de individuos o grupos específicos (22). Es decir, una prevención basada en lo biológico, que reduce la acción a la corrección funcional de riesgos, de forma aislada y fraccionada, para mantener la salud, desconociendo los DSS que inciden en la salud de la población.

Durante esta década, la prevención del VIH, en concordancia con la epidemiología tradicional, se caracterizó por un enfoque epidemiológico-conductual, en el cual, para contrarrestar la epidemia, los gobiernos implementaron políticas públicas centradas en la identificación de factores de riesgo individuales y conductas de riesgo de la transmisión del VIH, con énfasis en los HSH, antiguamente denominados población de homosexuales y bisexuales $(23,24)$.

En este periodo las estrategias de prevención dieron importancia a los procesos educativos, buscando cambios de comportamiento saludables; estrategias que mostraron poca efectividad dado que se dirigían casi exclusivamente a los HSH o "grupo de riesgo", generando respuestas sociales de estigma y discriminación y disminuyendo las posibilidades de una atención más cálida y de calidad. El riesgo a la infección fue concebido en términos de determinantes y conductas individuales, omitiendo elementos sociales, culturales, económicos y políticos que determinan la vulnerabilidad a la infección $(25,26)$, como lo demuestran los estudios de Liscano y Estrada (27), Manzano (28) y Tovar y Arrivillaga (29). A pesar de ello, de acuerdo con la literatura, la educación sigue vigente como una de las principales estrategias específicamente de prevención primaria.

24 En Colombia, desde el inicio de la infección en 1983, la prevención se orientó a generar cambios de comportamiento y estilos de vida. Las campañas masivas de comunicación e información fueron parte de las estrategías de prevención (24) y se consideraron útiles para crear en la población conciencia del VIH/SIDA, pero no para originar conductas preventivas propiamente dichas. Estas estrategias resultaron insuficientes, debido a que desconocieron cómo ciertas condiciones socieconómicas, culturales y políticas determinan la vulnerabilidad a la infección, como la pobreza y la mala gobernanza, entre otras. En consecuencia, la prevención de la infección debe incluir estrategias en el contexto de los DSS: atender la pobreza, el desempleo, la violencia de género, el conflicto armado, además de superar barreras socioculturales como el machismo y la homofobia $(9,18)$ y las barreras de acceso a la información, a los servicios de educación, salud y atención en salud integral con énfasis en la población más vulnerable, como mecanismos preventivos para controlar la infección por VIH.

Posteriormente, en la década de los noventa, la prevención del VIH, en correspondencia con el enfoque de la epidemiología crítica, enfatiza en los modos de vida o reproducción social y las condiciones de vida que afectan a la población. Por lo tanto, las acciones en salud se proyectan contra los procesos sociales que — se considera—destruyen la salud (PE) y a favor de los procesos que protegen tanto colectiva como individualmente la población (PS). En este enfoque las acciones se conciben de manera integral para responder a los procesos particulares, singulares y generales de los individuos y la población $(30,31)$, considerando que la PS y la PE se desarrollan en escenarios complejos de vida, con procesos multifactoriales, heterogéneos y cambiantes como son los diferentes contextos culturales, económicos, políticos, los estilos y modos de vida (clase social, edad, etnia, educación, empleo, entre otros) en los que se desarrollan las personas y los colectivos (31). 
La prevención del VIH en la década de los noventa, vinculada con la epidemiología crítica, se caracterizó por un enfoque antropológico y cultural. Trascendió de la perspectiva biomédica, fundamentada en el enfoque de riesgo y consideró la vulnerabilidad a la infección VIH, determinada por factores sociales y culturales que inciden en la conducta de los individuos $(23,24)$. Este enfoque incluyó todas las orientaciones sexuales para la prevención de los comportamientos de riesgo, de acuerdo con las singularidades y el entorno social de los individuos. Hizo énfasis en lo cultural y psicológico. Sin embargo, dio poca importancia a ciertos aspectos estructurales que determinan la propagación de la epidemia y que muchas veces están por fuera del control del individuo; por ello, pese a los avances en la prevención, no logró suficientes resultados en el control de la infección.

Un hecho importante durante esta década fue la aparición de la TAR en 1991. Con este avance científico se logró controlar el virus y prevenir la transmisión, y las personas mejoraron la posibilidad de supervivencia (32). No obstante, la implementación de la TAR generó el debate sobre el control de las nuevas infecciones VIH, por la toxicidad de los medicamentos ARV, la resistencia viral, los costos $(33,34)$, el acceso y adherencia al tratamiento, especialmente en las poblaciones más vulnerables.

En todo caso, el acceso a los ARV, de forma permanente, sostenida y con apoyo interdisciplinario, social y familiar (18), permitió brindar prevención secundaria a la persona con VIH, al mitigar los efectos de la infección en el afectado, y prevención primaria, al impedir que la persona afectada transmita la infección a sus eventuales contactos, interrumpiendo así la cadena de contagio y convirtiéndose en una estrategia fundamental de la prevención del VIH, pese a las críticas que se formulan por los costos financieros de su usoy los beneficios económicos que genera a la industria farmacéutica.
Colombia sigue los lineamientos mundiales para el control de la infección del VIH y, a partir de la Constitución Política de 1991 (35), derivó una serie de lineamientos políticos y normativos en torno a su problemática del VIH, como la Política Nacional de SSR de 1993, que centra su atención en el reconocimiento de los derechos sexuales y reproductivos (DSR) como DHy, tácitamente, vela por la libertad individual, incluyendo a la población más vulnerable a la infección VIH (36). Sin embargo, los miedos, los tabús y la represión para hablar de sexo obstaculizan reconocer y ejercer el derecho a los DSR.

De otra parte, los servicios de salud, vistos desde la perspectiva del mercado y la rentabilidad financiera, y no como derecho, pueden llegar a incidir en el incremento de la vulnerabilidad de los pobres y jóvenes a la infección por VIH, al restringírseles el acceso a los servicios de salud, lo que aumenta las posibilidades de adquirir la infección, desarrollar enfermedades oportunistas y el riesgo a la discapacidad y muerte (2).

Otros lineamientos normativos, como la Ley General de Educación —Ley 115 de 1994-, determinan "[...] desarrollar una sana sexualidad [...]” (37), en la que está implícita la prevención de las infecciones de transmisión sexual (ITS) y del VIH, cuya función es delegada a los educadores, quienes pueden o no estar preparados para responder a esta exigencia. La Ley 375 de 1997, de Juventud, decreta la garantía de la atención integral en los servicios de salud a los jóvenes (38), pero es preocupante el nivel de formación del personal de salud en el tema (39) y la claridad en la concepción de la prevención del VIH, a fin de garantizar dicha atención al joven.

E1 Decreto 1543 de 1997, específico en las orientaciones de prevención y control de VIH/ SIDA y otras ITS para Colombia (40), hace énfasis en la protección de los DH y resalta la 
educación como estrategia para la prevención. La normativa muestra interés por abordar la prevención del VIH desde distintos actores y sectores, como un compromiso político, con una serie de instrumentos normativos que vinculan al sector educativo como clave para la prevención del VIH en los jóvenes en general, pues existe evidencia científica de que la educación reduce el riesgo a la infección (41). Para ello, implementar lo normado desde el sistema educativo, los educadores requieren formación en el tema y condiciones laborales y sociales propicias para lograr impactos significativos en la prevención del VIH. En nuestro medio, las frecuentes protestas de los educadores (42) dan cuenta de su inconformismo, lo que puede convertirse en limitante para el éxito de las acciones de prevención de la infección por VIH, dirigida a los adolescentes escolarizados, en particular, $\mathrm{y}$ a los adolescentes, en general.

Autores como Arraes De Oliveira, Prado, Alves, Araujo, Souza, Matos. (43), Mantilla, Oviedo y Galvis (44), González, Savedra y Rojo (45), Cañas, Menéndez; Tápanes, Castillo, Abad. (46), Fadragas, Valdés y Fernández (47); Costa, Ferreira y Carvalho (48) señalan la importancia de la educación como estrategia para la prevención del VIH; asimismo, la Asamblea General de Naciones Unidas (Ungass), que propone incluir en los currículos temas relacionados con la salud sexual (SS) y la consejería dirigida a los niños y niñas, para disminuir la vulnerabilidad en los jóvenes (41) y promover la toma de decisiones y el ejercicio de una sexualidad responsable, sin dejar de lado acciones educativas específicas dirigidas a la población vulnerable al VIH.

El éxito de la prevención del VIH a través de estrategias de educación depende de la adecuada articulación intersectorial entre organismos internacionales y nacionales, la 26 empresa privada, los líderes religiosos, la familia, la escuela y los micropoderes del saber (prohibiciones, censuras, rechazos) en el interior de las organizaciones (49). Se debe resaltar la responsabilidad que compete a cada uno de los involucrados para la implementación de la educación como estrategia de prevención del VIH, lo cual se convierte en un verdadero desafío para su cumplimiento. Igualmente, existe la necesidad de enfatizar en acciones de intervención, desde todos los sectores, para la población con mayor grado de vulnerabilidad a la infección por VIH (50).

En estas dos primeras décadas de la infección del VIH, la prevención hizo énfasis en los cambios de comportamiento o estilos de vida de la población vulnerable, a través de la educación y la atención en los servicios de salud. La aparición de los ARV como estrategia para la prevención cambió el rumbo de la infección del VIH, la cual pasó de ser una enfermedad mortal a una enfermedad crónica controlable. Sin embargo, lo anterior no ha sido suficiente para el control de la infección, debido en parte a que el acceso a la educación, a los servicios de salud y a los ARV no está al alcance de toda la población. En consecuencia, se necesita un abordaje amplio de los DSS, con la participación y voluntad política de todos los actores participantes de la estrategia para lograr resultados positivos en la prevención de la infección.

Al inicio del siglo XXI surge el enfoque de prevención político-económico $(23,24)$, ligado a la medicina social, en la cual se concibe la $\mathrm{PE}$ en su contexto histórico, social y político, superando la lógica asistencial y de la causalidad de la enfermedad (51). De esta forma, se reconoce que los enfoques de prevención han avanzado de visiones individuales a perspectivas más incluyentes, estructurales y poblacionales, que sin duda deben incidir positivamente en la salud de la población.

Este enfoque plantea que las desigualdades económicas y sociales son la base de la epidemia del VIH y constituyen obstáculos para 
generar cambios de comportamiento saludables. Sustituye la idea de riesgo individual por los conceptos de vulnerabilidad social e individual y exclusión social. Los programas de esta orientación de la prevención están dirigidos a acciones en defensa de los derechos de las minorías y de las personas afectadas (23).

Se trata de una propuesta integral de prevención que incluye la atención y debe contribuir a transformar la estructura económica que relaciona al VIH/SIDA con la pobreza, los grupos marginales, la inequidad, el desempleo, el sexo por presión económica, el desplazamiento, los problemas de acceso a la educación, a los servicios de información y asistencia integral, que incrementan la vulnerabilidad a la infección de VIH y SIDA (2). Por ello, es necesario diseñar e implementar políticas públicas que actúen positivamente en los DSS y así lograr modificar los contextos y mejorar la calidad de vida de la población vulnerable a la infección.

En línea con lo anterior, la mayor parte de la población considerada vulnerable a la infección por VIH vive en países pobres, donde las desigualdades sociales son marcadas y las causas de la infección se asocian a los DSS. Esta situación impone a las estrategias de prevención del VIH promover acciones que respondan al contexto en el que viven las personas, avanzar del enfoque individual asistencialista a un enfoque de derecho integral, promover la cooperación social y la participación de los servicios de atención primaria específicos en VIH, como son los servicios de información, consejería, pruebas diagnósticas tempranas y disponibilidad de ARV. En suma, intervenir integralmente los DSS, para superar la inequidad y la exclusión social que favorecen la propagación de la infección.

Los países miembros de la OMS, en la búsqueda de un compromiso universal para alcanzar el desarrollo y superar la pobreza, acordaron los Objetivos de Desarrollo del
Milenio (ODM) 2000-2015, entre ellos el objetivo sexto en el cual se proponía "combatir el VIH/SIDA para el 2015 [...]” (52), y deberán continuar con la adopción de la estrategia del fast-track, ${ }^{3}$ con prioridad en la población vulnerable, con el compromiso mundial de finalizar el SIDA como problema de salud pública para el año 2030, en el marco de los Objetivos del Desarrollo Sostenible (ODS) (53).

Para este fin, de acuerdo con la OMS, se han considerado como principales métodos de intervención, utilizados de manera combinada para prevenir la infección del VIH: el uso correcto y sistemático de preservativos masculinos y femeninos; pruebas de detección rápidas; asesoría; circuncisión masculina voluntaria; tratamiento de ITS y tuberculosis, por ser un cofactor para la infección por VIH; tratamiento con ARV como prevención, profilaxis pre y posexposición; eliminación de la transmisión vertical del VIH; microbicidas para mujeres; reducción del daño en usuarios de drogas alucinógenas e intravenosas mediante el suministro de jeringas (54); intervenciones orientadas a cambios de comportamientos saludables; e intervención de situaciones estructurales como, por ejemplo, mejorar las condiciones de trabajo y el desempleo que definen la capacidad de consumo y el acceso a servicios de protección social para la prevención de la infección.

Para cumplir con el sexto ODM, "detener la epidemia [...]”, Colombia estableció la implementación de un plan intersectorial de prevención del VIH (52). Así, durante los años 2003-2013, la política de SSR priorizó el trabajo educativo con jóvenes e implementó el Modelo de Servicios de Salud Amigables para Adolescentes y Jóvenes, articulado con el sector educativo (36), servicios diferenciados que requieren superar la lógica del mercado de la salud ya mencionado, facilitar el acceso

3 Estrategia de respuesta rápida (53). 
a las poblaciones más afectadas y garantizar mayor divulgación para que contribuyan a la prevención de la infección.

Recientemente, con la expedición de la Resolución 1841 de 2013, se adopta el Plan Decenal de Salud Pública (PDSP) (20122021), en el cual se encuentran la "sexualidad, los DSR y la prevención del VIH/SIDA" como prioridad que debe promover acciones sectoriales, transectoriales y comunitarias, para la afectación de los DSS que inciden en el las ITS-VIH/SIDA, con énfasis en poblaciones en contextos de mayor vulnerabilidad (55). El PDSP también propone garantizar el acceso a los servicios de prevención y atención integral en salud para el control de la infección del VIH (56).

En este marco normativo, la prevención delVIH concentra la inversión en la atención, diagnóstico y tratamiento de la infección. No obstante, el alto costo de los ARVy de los servicios integrales actúa como barrera que limita el acceso a los servicios por parte de la población afectada y vulnera su derecho a la salud. De hecho, en el año 2014, en Colombia se interpusieron 118281 acciones de tutela en las cuales se invoca el derecho a la salud, 901 de ellas específicas para VIH y SIDA $(57,58)$. Estas restricciones entorpecen las estrategias de prevención de la infección y perjudican la calidad de vida y la economía de la persona afectada y su familia (59).

El enfoque de prevención del VIH basado en el manejo farmacológico plantea, empero, simplificar el tratamiento, generalizar las pruebas y el acceso universal a los ARV, considerando que el balance riesgo-beneficio permite su uso, debido a que los beneficios superan ampliamente los riesgos, de tal manera que las críticas generadas por el costo de los medicamentos ARV $(33,34)$ pueden ser mitigadas con la adopción de políticas y estrategias de control de precios que permitan asumir su costo y el acceso universal.
Este abordaje, basado en la atención en salud y la terapia con ARV, para algunos autores $(36,60)$ responde a la visión del modelo biomédico convencional, reconocido como reduccionista de la enfermedad, que deja en segundo plano las acciones de prevención primaria clásica como la promoción de comportamientos sexuales seguros y responsables, a través de la información y la educación, y los nuevos enfoques que buscan intervenir los DSS. Sin embargo, la prevención centrada en la terapia con ARV brinda prevención primaria para aquellos que, en ejercicio de sus DSR (56), asumen conductas de riesgo y se exponen al contagio de la enfermedad. A este respecto, los organismos internacionales y autores como Peretti-Watel, Spire, Obadía y Moatti (61) reconocen el estrecho vínculo entre la prevención y el acceso a los ARV y la necesidad urgente de suministrar tratamiento de manera universal.

La prevención del VIH mediante la atención con TAR, como nueva concepción de prevención, ha logrado resultados significativos como la reducción de la transmisión vertical y los casos notificados por sida $(62,63)$. Pese a ello, en nuestro país el aumento de nuevos casos notificados con VIH entre los años 2013 y 2014 (10) muestra que la cobertura y el acceso a los ARV no son suficientes para el control de la epidemia. Se necesita empoderar a la población sobre sus derechos, fortalecer y acercar las acciones de promoción y prevención a la población más vulnerable al VIH y diseñar y adoptar políticas y estrategias que garanticen el acceso universal a los ARV, como elemento clave en la prevención por su acción en la interrupción de la cadena de transmisión de la infección.

Con todo lo anterior, se puede entender la PE como la posibilidad de evitar la aparición de enfermedades y lesiones, mediante acciones o estrategias que actúan sobre las condiciones que favorecen la aparición de la lesión o de la enfermedad. Su propósito es disminuir el 
riesgo de enfermar. En el caso de las estrategias de prevención del VIH, las acciones tienen que avanzar del enfoque asistencialista y las demandas de servicios integrales a políticas que promuevan el desarrollo humano, social y económico. Estas políticas deberán enfatizar en la promoción de estilos y modos de vida saludable, los comportamientos sexuales seguros, el acceso a los ARV e incidir positivamente en las condiciones sociales, económicas, culturales y políticas que favorecen la aparición de la infección del VIH.

En la actualidad, la prevención sigue vigente, con una mayor comprensión de la realidad social, con enfoques más estructurales para el control de la infección del VIH. Por lo tanto, desde la perspectiva de los DSS, se requieren propuestas de vanguardia que promuevan:

i) El fortalecimiento de la articulación de las acciones emprendidas por los diferentes sectores y actores involucrados, con participación de la población afectada, para la prevención del VIH; ii) la divulgación de información para la prevención del VIH en los medios de comunicación, de manera sostenida y en horarios de mayor audiencia (radio, televisión) y en eventos masivos (cine, eventos deportivos y artísticos), donde los mensajes logren crear conciencia de la infección en la población; iii) el fortalecimiento de la movilización y la participación social a través de la formación y el empoderamiento de las organizaciones civiles y de jóvenes como principal red de apoyo con sus pares, ${ }^{4}$ para la prevención de la infección VIH en los diferentes ámbitos de la vida cotidiana (escuela, familia, vecindario, espacios recreativos y laborales); iv) el diseño y la implementación de políticas encaminadas a reducir el costo de los ARVy los insumos requeridos para el diagnóstico y el seguimiento

4 Se refiere a "una persona de la misma posición social que otra; que pertenece al mismo grupo social, en particular respecto a la edad, el nivel de estudios o la situación social" (64). a la población afectada o vulnerable, de tal manera que su cobertura sea universal y sus costos no continúen actuando como barreras de acceso a esta estrategia de prevención primaria; v) el fortalecimiento de políticas orientadas a ofrecer igualdad de oportunidades: vida digna, trabajo digno, acceso universal a la educación, a la recreación y a servicios de salud de calidad.

Paralelamente, para controlar la infección, el país, por tener una epidemia concentrada, debe diseñar e implementar programas innovadores, con enfoque de derecho y vulnerabilidad adecuados al contexto y orientados a las poblaciones consideradas de mayor vulnerabilidad, que incluya:

i) el fortalecimiento de los servicios integrales de atención VIH dirigidos a las/los trabajadores sexuales; ii) reforzar el acceso a la educación e información específica para esta población; iii) kits asépticos para los usuarios de drogas inyectables, o sustitución de sustancias psicoactivas, incluyendo la PPL; iv) ampliar centros comunitarios para los transexuales y fortalecer los servicios integrales de atención VIH; v) fortalecer y generalizar la divulgación a través de redes sociales y llevar las acciones a sitios públicos; vi) ofrecer la prueba autodiagnóstica; vii) facilitar el acceso a los ARV de forma inmediata, una vez se tiene el diagnóstico o cuando se expuso al riesgo; viii) mayor cobertura y racionalidad financiera que garantice la atención integral; ix) ofrecer y facilitar la asesoría jurídica que garantice los derechos de acceso a los servicios integrales, en caso necesario.

Estas acciones específicas de prevención del VIH para la población de mayor vulnerabilidad son deseables y solo serán posibles si se precisan en los planes de beneficios establecidos en el Sistema General de Seguridad Social en Salud (SGSSS) y, además, si la financiación se garantiza con los recursos destinados por 
la nación para tal fin y a través de políticas de control de precios que superen la barreras de acceso por las restricciones financieras del sistema de salud, en la lógica de la rentabilidad de la salud como mercancía; por el tipo de aseguramiento y por el estigma y la discriminación que sufre la población más vulnerable, en el marco de la garantía del derecho a la salud.

\section{Realidades}

La Política de Prevención del VIH en Colombia sigue los lineamientos internacionales establecidos por la OMS, la OPS, Onusida y Ungass, quizá sin conocer a profundidad las experiencias locales de atención, las particularidades sociales, económicas y culturales de nuestro país, lo que puede dar lugar a una adaptación descontextualizada (59). Si bien se reconocen los esfuerzos de estos organismos para dar respuestas oportunas y apropiadas en defensa de los derechos de las personas más vulnerables al VIH y el SIDA, son necesarios mayores esfuerzos financieros para superar las restricciones económicas que impiden el acceso universal a la prevención del VIH y la atención integral (asesoría, pruebas rápidas, acceso a TAR), con énfasis en la población más vulnerable.

La prevención del VIH, desde la perspectiva de la salud pública, reconoce los avances científico-tecnológicos, la atención integral y el establecimiento de políticas de escala mundial y nacional; pero, pese a ello, los resultados no son los ideales, ya que siguen infectándose y muriendo personas, con predominio de los jóvenes, quizá como resultado de la pobreza, la exclusión social, la ignorancia, el estigma, la discriminación (65) y las múltiples barreras para el acceso a la atención integral en nuestro sistema de salud. los DSR para toda la población, en ocasiones son vulnerados por los agentes del sistema de salud y la sociedad. En relación con el VIH, por temor e ignorancia de los profesionales, lo que se puede traducir en prácticas discriminatorias (4); además, por la inestabilidad laboral y la rotación del personal de salud, situación que no garantiza la oportunidad, la continuidad y la integralidad de la atención, incluidos los servicios de prevención de ITS/ VIH, y que, por el contrario, fragmenta las acciones de prevención.

Sumado a lo anterior, los servicios de salud "no siempre están preparados para brindar una prevención centrada en la atención amigable, prevalecen ideas moralistas, religiosas y a veces poco científicas desde las instituciones y algunos profesionales de la salud" (36). Este escenario, aliado a las restricciones financieras y sociales, a la precaria información e inadecuada atención, se convierte en barrera para acceder a la prevención de la infección y a tratamientos oportunos con ARV, lo que favorece la aparición de nuevas infecciones por VIH.

\section{Brechas}

Si bien es cierto, Colombia tiene una cobertura de afiliación en salud del $92 \%$ de la población (62), aún se identifican dificultades para la prevención del VIH por las barreras de acceso a los servicios integrales, por falta de aseguramiento, acentuado en la población considerada de mayor vulnerabilidad social, "población pobre", sin estabilidad laboral y sin ingresos; además de las inequidades geográficas (62), situación que determina el acceso, la calidad y la continuidad de los servicios de prevención y tratamiento del VIH.

En el país es necesario promover el acceso universal a los TAR, como estrategia clave de prevención, restringido en la población de mayor vulnerabilidad por las razones expuestas, y cerrar la brecha entre quienes tienen 
acceso a los servicios de prevención del VIH, tratamiento y apoyo, y los que no lo tienen; además, fortalecer la accesibilidad al diagnóstico y tratamiento rápido para mejorar su calidad de vida e incrementar la supervivencia, así como contribuir a contener la epidemia en un mediano plazo.

En el mismo sentido, aunque la educación y la información se consideran fundamentales como estrategias para la prevención del VIH, capaces de promover comportamientos sexuales seguros y responsables (66), resultan insuficientes por los temores de los educadores y padres a tratar temas de sexualidad; las restricciones impuestas por líderes religiosos; la dinámica de la escuela actual, subordinada a las exigencias del sistema económico ( (67) y la adaptación de currículos de otros contextos que no responden a las necesidades particulares del país ((64). Factores todos que afectan la implementación de estrategias de educación en general y de la prevención del VIH en particular, y se suman a la inequidad en el acceso a la educación, especialmente de la población vulnerable.

Por lo tanto, la educación para la prevención del VIH debe ser un proceso flexible, con enfoque de derecho, que estimule la participación activa de los involucrados, reconozca las diferencias culturales y promueva procesos de reflexión y construcción colectiva del conocimiento de los participantes, quienes se convertirán en multiplicadores entre sus pares (68) para la toma de decisiones responsables e informadas como estrategia para la prevención de la infección.

\section{Conclusiones}

Las estrategias de prevención del VIH tienen que avanzar del enfoque asistencialista y las demandas de los servicios integrales a políticas que promuevan el desarrollo humano, social y económico $(2,69)$ a mediano y largo plazo, en las cuales la población pueda participar de manera democrática en las decisiones que la afectan, que busquen el mejoramiento de las condiciones de vida, el acceso a la educación y al trabajo digno y disminuyan las brechas sociales (2). Asimismo, estas estrategias deben responder a las necesidades particulares, de género y orientación sexual, posición social y a las particularidades culturales del contexto al que se pertenece. En síntesis, garantizar el acceso real y con calidad a los servicios de salud e intervenir positivamente en los DSS.

A pesar de los logros y fracasos enmarcados en los lineamientos políticos del ámbito internacional y nacional, persisten vacíos políticos y normativos; fallas en la implementación y despliegue de las políticas de salud y educación para la prevención del VIH; brechas entre los sectores para el trabajo integral; inequidad de los sectores salud, educación y otros importantes sectores; escaso apoyo social; deficiente información y divulgación para la prevención de la infección en los medios que, por el contrario, envían mensajes que estimulan a optar por comportamientos, frente al cuerpo y sexualidad, en contravía de las acciones educativas de PS y PE para el control de la infección.

Finalmente, la prevención del VIH se convierte en un reto para la salud pública, pues le exige continuar ampliando y validando el conocimiento desde la investigación. Esto le permitirá reorientar, desde su conceptualización, la manera de operacionalizar y redireccionar políticas públicas, planes, programas y estrategias en este campo, con énfasis en la población más vulnerable. Pero, además, en esta búsqueda por la igualdad de oportunidades y el control de la infección, no se puede olvidar que es la población, en general, la que se encuentra en riesgo de adquirir y desarrollar la infección del VIH. 


\section{Referencias bibliográficas}

1. Cardona-Arias J. Representaciones sociales de calidad de vida relacionado con la salud en personas con VIH/SIDA. Rev Salud Pública Universidad de Antioquia. 2010;12(5):765-76 [internet]. Disponible en: http://www.scielosp.org/pdf/rsap/v12n5/ v12n5a07.pdf

2. Lopera M, Bula JI. Impacto socioeconómico del VIH en las familias: Fenómeno olvidado en la política de atención integral. En: Arrivillaga M, Useche B. editores. Sida y sociedad. Bogotá: Aurora; 2011.

3. Rosado Pérez G, Rivera Reyes G, Larrieux Villanueva V, Torres G, Betancourt Díaz E, Villaruel A, et al. Estigma y VIH/SIDA entre padres/madres y adolescentes puertorriqueños. Revista Puertorriqueña de Psicología. 2016;27(1):134-45.

4. Aristizábal C. Estigma y discriminación frente a las personas con VIH como fuente de vulnerabilidad e inequidad en la atención en salud. En: Arrivillaga M, Useche B, editores. Sida y sociedad. Bogotá: Aurora; 2011.

5. Organización de las Naciones Unidas sobre el VIH/ SIDA. Onusida. Ficha Informativa. Estadística How AIDS changed everything 2014. [internet] marzo de 2015. Disponible en: http://www.unaids.org/es/ resources/documents/2015/20150714_factsheet

6. Pérez C. A 30 años del primer paciente diagnosticado con VIH en Chile. Académico. Boletín Informativo Pontificia Universidad Católica de Chile. 2014 agosto [acceso: 23 de septiembre de 2015]. Disponible en: http://medicina.uc.cl/ agosto-2014/a-30-anos-del-primer-paciente-diagnosticado-con-vih-en-chile-academico-uc-participo-de-congreso-mundial-de-sida-en-australia

7. Méndez C, Warner K. Mujeres VIH y SIDA y su adherencia: Un análisis de la Política Pública desde los determinantes de la salud. Cadernos Ibero-Americanos de Direito Sanitário. Brasilia. 2014 julio/septiembre;3(2):30-54 [acceso: 9 de octubre de 2015] [internet]. Disponible en: http://www. cadernos.prodisa.fiocruz.br/index.php/cadernos/ article/view/8

8. Useche B, Cabezas A. El modelo neoliberal en los tiempos del SIDA. Revista digital Envío Universidad Centroamericana UCA Nicaragua. 2005 agosto;285 [internet]. Disponible en: http://www.envio.org.ni/ articulo/3008

9. García R. ODM6. Combatir el VIH SIDA, la malaria y otras enfermedades. En: Corredor C, editor. ODM. Los objetivos de desarrollo del milenio. Bogotá: Universidad Nacional de Colombia; 2010.

10. Ministerio de Salud y Protección Social. MSPS. Informe Nacional GARPR. Seguimiento de la Declaración de compromiso sobre el VIH/Sida. 2014 [internet]. Disponible en: http://files.unaids.org/en/ dataanalysis/knowyourresponse/countryprogressreports/2014countries/COL_narrative_report_2014. pdf

11. Secretaría Distrital de Salud. Mecanismos de Coordinación del País. MCP Colombia. Fondo Mundial de Lucha contra el Sida. Resultados comportamiento sexual y prevalencia de la infección VIH población habitante de calle. 2012 [internet]. Disponible en: http://www.saludcapital.gov.co/DSP/Informes $\% 20$ Trimestrales\%20VIH/Prevalencia\%20VIH\%20 Habitante\%20Calle\%202012.pdf

12. Organización de las Naciones Unidas. Onusida. Informe sobre la epidemia mundial SIDA [internet] 2014 Julio [acceso: 12 de octubre de 2014]. Disponible en: http://www.unaids.org/es/resources/ presscentre/pressreleaseandstatementarchive/2014/ july/prgapreport

13. Cuellar N. Instituto Nacional de Salud. VIH/SIDA Informe del comportamiento en la notificación del VIH-SIDA periodo epidemiológico 12 del año 2014 [acceso: 7 de febrero de 2015] [internet]. Disponible en: http://www.ins.gov.co/lineas-deaccion/SubdireccionVigilancia /Informe\%/VIH\%20Periodo\%20 XII\%202014.pdf

14. Profamilia, Ministerio de salud y Protección Social MSPS, Bienestar Familiar ENDS. Encuesta Nacional de Demografía y Salud. ENDS. Colombia: 2010.

15. Ministerio de salud y Protección Social MSPS. Boletín Epidemiológico. Situación del VIH/SIDA [internet] 2013. Disponible en: https://www.minsalud.gov.co/Documentos\%20y\%20Publicaciones/ BOLETIN\%20EPIDEMIOLOGICO\%20VIH\%20 1983-2012.pdf

16. Organización Mundial de la Salud OMS. Programa de la OMS sobre el VIH/SIDA. Fortalecer el sector sanitario para alcanzar el acceso universal a la prevención, tratamiento y atención del VIH. 2007. Disponible en: http://www.who.int/hiv/mediacentre/ Who_we_are_A4_es.pdf

17. Organización Panamericana de la Salud, Organización Mundial de la Salud. Atención Primaria en Salud y los Objetivos del Milenio. 2012 [acceso: 5 de julio de 2015]. Disponible en: http://www.paho.org/ blogs/chile/?p=153

18. Aristizábal C. ODM6. La infección por VIH: enfermedad cultural y de la pobreza. En: Corredor C, editor. ODM: Los objetivos de desarrollo del Milenio. Bogotá: Universidad Nacional de Colombia Antropos; 2010.

19. Kottow M. Bioética en salud pública: protección y confianza en VIH. Revista Chilena Salud Pública. 2007;11(2):83-88 [internet] Disponible en: file://C:/ Users/marta/Downloads/8201-18594-1-PB.pdf

20. Bayer R, Fairchild A. Changing the paradigm for HIV Testing. The end of exceptionalism. The New England Journal of Medicine [internet]. 2006; 355(647-649). doi: 10.1056/NEJMp068153. Disponible en: http://www.nejm.org/doi/full/10.1056/ NEJMp068153?query $=$ TOC\&\#R2

21. Bayer R, Kirp D. Reflexión conclusiva en defensa del voluntarismo. En: Ferrer J. Sida y bioética de la autonomía a la justicia. Madrid, 1997 [acceso: 2 de diciembre de 2015]. Disponible en: https://books.google. com.co/books? id = PgE5M5oEj8MC\&pg $=$ PA7\&d$\mathrm{q}=$ voluntarismo + vih $+\mathrm{a} \&$ source $=$ gbs_selected_pa$\operatorname{ges} \& \operatorname{cad}=2 \# \mathrm{v}=$ onepage $\& \mathrm{q}=$ voluntarismo $\% 20$ vih\%20a\&f=false

22. Díaz S, Rodríguez L, Valencia A. Análisis de publicaciones en promoción de la salud: una mirada a las tendencias relacionadas con prevención de 


\section{PREVENCIÓN DE LA INFECCIÓN POR EL VIRUS DE LA INMUNODEFICIENCIA HUMANA (VIH) EN COLOMBIA: BRECHAS Y REALIDADES}

la enfermedad. Gerencia y Políticas Públicas de Salud. 2015 marzo;14(28):32-47 [internet]. Disponible en: http://dx.doi.org/10.11144/Javeriana. rgyps18-28.apps

23. Estrada JH, Vargas L. Invisibles y excluidos: la infección VIH/SIDA entre hombres que tienen sexo con hombres (HSH). En: Arrivillaga M, Useche B. Sida y sociedad. Bogotá: Aurora; 2011. p. 115-59.

24. Cáceres C. Intervenciones para la prevención de VIH e ITS em América Latina y Caribe: uma revisión de la experiência regiona. Cad. Saúde Pública. 2004 Nov-Dez;20(6):1468-85 [internet]. Disponible en: http:/www.scielo.br/pdf/csp/v20n6/04.pdf

25. Rubiano Y, Saldarriaga J. Jóvenes y VIH/SIDA: enfoques y perspectivas en investigación. Index Enfermería. 2011 junio; 20(1-2): 76-80 [acceso: 2 de febrero de 2016]. Disponible en: http://scielo.isciii. es/scielo.php?pid=S1132-12962011000100016\&script $=$ sci_arttext

26. Uribe F. Limitaciones conceptuales en epidemiología para la clasificación del comportamiento sexual masculino en el contexto de la epidemia de VIH en México. Andamios. 2008;4(8):213-42 [internet]. Disponible en: http://www.scielo.org.mx/scielo.php? script=sci_arttext\&pid=S1870-00632008000100009\&1n$\mathrm{g}=\mathrm{es} \& \mathrm{t} \operatorname{lng}=\mathrm{es}$

27. Liscano Y, Estrada JH. Exploración y análisis a los determinantes sociales estructurales e intermedios de la pandemia del VIH/SIDA. Acta Odontológica Colombiana. 2015 enero-junio;5(1):117-31 [acceso: 2 de octubre de 2016]. Disponible en: http://www. revistas.unal.edu.co/index.php/actaodontocol/article/view/52285/52029.

28. Manzano J. VIH/SIDA y determinantes sociales de la salud. 2012. Disponible en: http://jesusmanzano.es/2012/11/vihsida-y-determinantes-sociales-de-la-salud.html

29. Tovar LM, Arrivillaga M. VIH/SIDAy determinantes sociales estructurales en municipios del Valle del Cauca Colombia. Revista Gerencia y Políticas en Salud. 2011;10(21):112-23 [acceso: $1 .^{\circ}$ de octubre de 2016]. Disponible en: http://rev_gerenc_polit_salud. javeriana.edu.co/vol10_n_21/estudios_3.pdf

30. Breilh J. Epidemiología crítica. Ciencia emancipadora e interculturalidad. Buenos Aires: Lugar; 2003.

31. Epidemiología crítica latinoamericana: raíces, desarrollos recientes y ruptura metodológica. En: Morales C y Eslava J. Tras las huellas de la determinación. Bogotá: Universidad Nacional; 2015.

32. Herrera C. Sida, ahora una enfermedad controlable. UN Periódico. 2008 marzo. Disponible en: http:// historico.unperiodico.unal.edu.co/ediciones/109/09. html

33. Useche B. TLC Colombia-Estados Unidos. Propiedad intelectual, patentes y acceso a medicamentos genéricos. Deslinde. 2007 mayo;47:52-69 [internet]. Disponible en: http://cedetrabajo.org/wp-content/ uploads/2012/08/41-7.pdf

34. Rossi F. Medicamentos para el VIH y el SIDA: Hablamos de salud o hablamos de negocios. En: Arrivillaga M, Useche B, editores. Sida y sociedad. Bogotá: Aurora; 2011. p. 45-85.
35. Constitución Política de Colombia. Gaceta Constitucional N. ${ }^{\circ}$ 127, Bogotá, 1991.

36. Rodríguez C. Las y los jóvenes como sujetos de derechos y los derechos sexuales y reproductivos. En: Morales C. Jóvenes, sexualidad y politícas. Cuadernos del Doctorado 14. Bogotá: Universidad Nacional de Colombia; 2011.

37. Congreso de la República de Colombia. Ley General de Educación de Colombia. Ley 115 de 1994 Diario oficial 41.214, 8 de febrero de 1994.

38. Congreso de la República de Colombia. Ley de la Juventud, Ley 375, 4 de julio de 1997. Disponible en: http://www.mineducacion.gov.co/1621/articles-85935_archivo_pdf.pdf

39. Uribe A, Orcasita L. Evaluación de conocimientos, actitudes, susceptibilidad y autoeficacia frente al VIH/SIDA en profesionales de la salud. Avances en Enfermería. 2011 julio-diciembre; 29(2):271-84.

40. Ministerio de la Protección Social. Colombia. Decreto 1543. Por el cual se reglamenta el manejo de la infección por el Virus de Inmunodeficiencia Humana (VIH) [internet], 12 de junio de 1997 [acceso: 15 de junio de 2014]. Disponible en: http://www.icbf.gov. co/cargues/avance/docs/decreto_1543_1997.htm

41. Aggleton P, Clarke D. Life skills-based HIV education. United Nation Educational, Scientific and Cultural Organization (Unesco), 2012. Disponible en: http:// unesdoc.unesco.org/images/0021/002178/217866e.pdf

42. Tema de evaluación docente acuerdo con maestros. Paro continúa. El Tiempo [internet]. 2014 mayo 15 [acceso: 15 de marzo de 2015]. Disponible en: http://www.eltiempo.com/estilo-de-vida/educacion/ paro-de-maestros-en-colombia/13990705

43. Arraes De Oliveira C, Prado Palos MA, Alves Barbosa M, Araujo Teles S, Souza de MM, Mattos de MA. Masculinity, vulnerability and prevention of STD/HIV/ AIDS among male adolescents: social representations in a land reform settlement. Rev. Latino-Am. Enfermagem [internet]. 2013;21(6):1266-73 [ acceso: 7 de marzo de 2015]. doi:10.1590/0104-1169.3059.2363

44. Mantilla B, Oviedo MP, Galvis DC. Programas de educación sexual y reproductiva: significados asignados por jóvenes. Colombia. Hacia la Promoción de la Salud. 2013;18(1):97-109 [acceso: 12 de febrero de 2015] [internet]. Disponible en: http://www.scielo. org.co/pdf/hpsal/v18n1/v18n1a08.pdf

45. González T, Saavedra J, Rojo P. Adolescencia: Peculiaridades paso a adultos. Revista de Enfermedades Infecciosas en Pediatría [internet]. 2012;25(102):201-5 [acceso: 12 de marzo de 2015]. Disponible en: http://new.medigraphic.com/cgi-bin/ resumen.cgi? IDARTICULO $=43118$.

46. Cañas LC, Menéndez CR; Tápanes FT, Castillo FR, Abad LY. Intervención educativa sobre el VIH con adolescentes de la Escuela Militar Camilo Cienfuegos de Arroyo Arenas. Revista Cubana de Medicina Tropical Cuba. 2012;64(1):43-48.

47. Fadragas A, Valdés M, Fernández M. Caracterización del nivel de información sobre ITS/VIH/ SIDA en adolescentes. Revista Cubana de Medicina General Integral. 2012;28(1):15-25 [acceso: 15 de febrero de 2015]. Disponible en: http:// scielo.sld.cu/scielo.php?script $=$ sci_arttext $\&$ pi$\mathrm{d}=$ S0864-21252012000100003\&lang $=-\mathrm{pt}$ 
48. Costa M, Ferreira R, Carvalho HM. Intervenções de educação sexual em adolescentes: uma revisão sistemática da literatura. (Portuguese). Revista de Enfermagem Referência [internet]. 2013 julio;(10):125-34 [acceso: 5 de marzo de 2015]. Disponible en: http:// www.scielo.mec.pt/scielo.php?script $=$ sci_arttext\&pid=S0874-02832013000200015

49. Foucault M. Historia de la sexualidad. La voluntad del saber. México: Siglo XXI; 2011.

50. Organización de las Naciones Unidas. Onusida. Fast-track. Ending the AIDS epidemic by 2030 [internet]. 2015. Disponible en: http://www.unaids.org/ sites/default/files/media_asset/JC2686_WAD2014report_es.pdf

51. Eslava J. Promoción y prevención en el sistema de salud en Colombia. Rev Salud Pública. 2002;4(1):1-12 [internet]. Disponible en: http:// www.scielo.org.co/scielo.php?script $=$ sci_arttext\&pi$\mathrm{d}=\mathrm{S} 0124-00642002000100001 \& \operatorname{lng}=\mathrm{en} \dot{\mathrm{Q}} \mathrm{nrm}=$ iso

52. República de Colombia, Departamento de Planeación Nacional. Conpes social. Metas y estrategias de Colombia para el logro de los Objetivos de Desarrollo del Milenio 2015 [internet]. 2005. Disponible en: https://www.minambiente.gov.co/images/normativa/ conpes/2005/Conpes_0091_2005.pdf

53. United Nations Program on HIV/AIDS. Unaids. Fast track - Ending the AIDS epidemic by 2030 [internet]. 2015. Disponible en: http://www.unaids. org/sites/default/files/media_asset/JC2686_WAD2014report_en.pdf

54. Organización Mundial de la Salud. OMS. VIH/sida. Nota descriptiva N. ${ }^{\circ} 360,2015$ [internet]. Disponible en: http://www.who.int/mediacentre/factsheets/ fs $360 / \mathrm{es} /$

55. Ministerio de Salud y Protección Social (MSPS). Plan Decenal de Salud Pública PDSP 2012-2021. Bogotá: MSPS; 2012.

56. Ministerio de Salud y Protección Social (MSPS). Política Nacional de Sexualidad. Derechos Sexuales y Derechos Reproductivos. 2014 [internet] [acceso: 9 de octubre de 2015]. Disponible en: https://www. minsalud.gov.co/sites/rid/Lists/BibliotecaDigital/ RIDE/DE/LIBRO\%20POLITICA\%20SEXUAL\%20 SEPT\%2010.pdf

57. Defensoría del Pueblo. Colombia. La tutela y los derechos a la salud y a la seguridad social. 2014 [internet] [acceso: 20 de febrero de 2016]. Disponible en: http:// www.defensoria.gov.co/public/pdf/LatutelaylosderechosalaSalud.pdf

58. Corte Constitucional, Colombia. Sentencia T-920/13 Derecho a la salud. 2013 [internet]. Disponible en: http://www.corteconstitucional.gov.co/RELATORIA/2013/T-920-13.htm

59. Arrivillaga M, Alzate ML, Useche B. Políticas públicas. Sistema de salud y mujeres con VIH/SIDA en
Colombia. Rev Gerencia y Políticas de Salud [internet]. 2009;8(16):58-71. Disponible en: http://www. scielo.org.co/pdf/rgps/v8n16/v8n16a04.pdf

60. Rodríguez MM, Echemendía TB. La prevención en salud: posibilidad y realidad. Rev Cubana Hig Epidemiol [internet]. 2011;49(1):135-50 [acceso: 30 de junio de 2015]. Disponible en: http:// scielo.sld.cu/scielo.php?script = sci_arttext\&pi$\mathrm{d}=\mathrm{S} 1561-30032011000100015 \& \operatorname{lng}=\overline{\mathrm{es}}$

61. Peretti-Watel P, Spire B, Obadía Y, Moatti JP. Discrimination against HIV-infected and the spread of HIV: some evidence from France. Journal Plos One [internet]. 2007 mayo 2; 2(5):e411. Disponible en: http://www.ncbi.nlm.nih.gov/pmc/articles/ PMC1853240/

62. Ministerio de Salud y Protección Social (MSPS), Onusida - Grupo Temático para Colombia. Plan Nacional de Respuesta ante las ITS VIH/SIDA. 2014-2017 [internet]. Disponible en: http://www. nationalplanningcycles.org/sites/default/files/planning_cycle_repository/colombia/plan-nacional-respuesta-its-2014-2017.pdf

63. Organización Panamericana de la Salud, Organización Mundial de la Salud, Unicef. Eliminación de la transmisión maternoinfantil del VIH y la sífilis en las Américas. 2015 [internet]. Disponible en: http://iris.paho.org/xmlui/bitstream/handle/123456789/18373/9789275318706_esp.pdf?sequence $=3 \&$ isAllowed $=\mathrm{y}$

64. Vega R. La universidad de la ignorancia. Bogotá: Oceansur; 2015.

65. Alzate ML. Treinta años de una pandemia que mueve el mundo. Bogotá: Universidad Nacional de Colombia, Cuadernos del Doctorado N. ${ }^{\circ}$ 15; 2012.

66. Sam-Soto S, Osorio-Caballero M, Rodríguez-Guerrero RE, Pérez-Ramírez NP. Comportamiento sexual y anticoncepción en la adolescencia. Acta Pediátrica de México [internet]. 2014;35(6):490-8 [acceso: 12 de marzo de 2015]. Disponible en: http:// www.medigraphic.com/pdfs/actpedmex/apm-2014/ apm146h.pdf

67. Suárez H, Valencia A. Educación y democracia: un campo de combate. Estanislao Zuleta. Bogotá: Tercer Milenio; 1995.

68. Organización de las Naciones Unidas. Onusida. Peer education and HIV/AIDS: concepts, uses and challenges. 2000 [internet]. Disponible en: http:// www.unaids.org/sites/default/files/media_asset/ jc291-peereduc_es_0.pdf

69. ONU, Asamblea General. Declaración política sobre elVIH y el SIDA: Intensificación de nuestro esfuerzo para eliminar el VIH y el sida. 8 de julio de 2011 [internet]. Disponible en: http://www.unaids.org/sites/ default/files/sub_landing/files/20110610_UN_ARES-65-277_es.pdf 